\title{
Simulation Study on Deposition Downstream Floodgates in Estuary
}

\author{
X.M. WANG \& L. DING \& X.Z. ZHANG \& X.P. DOU \& J. JIAO \\ Nanjing Hydraulic Research Institute, Key Lab of Port \\ Waterway and Sedimentation Engineering of the Ministry of Transport, Nanjing, China
}

KEYWORD: Estuary with floodgate; Numerical model; Sediment transport; Siltation characteristics.

\begin{abstract}
Siltation in the rivers downstream of floodgates is serious in China, making the discharge capacity of rivers decrease obviously and threatening the flood control in upper rivers. Tidal wave deformation is the dynamic characteristic of downstream floodgates, sediment from the sea is the main source of deposition downstream floodgate and unbalanced sediment transportation is the basic model. In the planning and design of floodgate situation choice, coastal deposition and direction of downstream floodgate should be considered. A 2-D numerical model of tide and sediment transport is established to simulate the distribution of sediment siltation downstream of the floodgate, and several conclusions are obtained in this paper which can provide some theoretical basis for the prediction and analysis of the siltation characteristics in lower reach caused by floodgates in estuaries.
\end{abstract}

\section{INTRODUCTION}

In order to defend against tides for the development of agricultural production, lots of floodgates have been built in coastal areas of China since 1950s, with the number of about 300 now. Siltation in the rivers downstream of floodgates is very serious in China and according to statistics, among the 58 floodgates with the drainage flow of over $100 \mathrm{~m}^{3} / \mathrm{s}$ in Jiangsu Province, 5 almost are silted up, 15 are in severe siltation and the other 38 are relatively less silted. Besides, in the 35 estuaries with floodgates constructed in Haihe River basin, there are 22 in serious siltation, where the upper and lower reaches are universally silted, causing the river drainage capacity reduced by $60 \%$. Siltation in the rivers downstream of floodgates makes the discharge capacity of rivers decrease obviously and threatening the flood control in upper rivers, and influencing the agricultural production. Therefore, it is vital to do research about siltation downstream of floodgates.

Study on siltation downstream of floodgates in China began with research on Sheyang Estuary by G.R. Dou (1963), showing that tidal wave deformation was main factors leading to sedimentation downstream floodgate. Tidal wave deformation and reflection usually occur under the influence of river morphologic resistance when it comes into the estuary, which will be more evident in the estuaries where floodgates have been built. Tidal wave will be reflected fully by floodgate, and tidal wave properties change from progressive wave to standing wave, there being a significant phase difference between hydrographs of flow velocity and tide level. Besides, high tide level rises while low tide level goes down, duration of flood tide is shortened and mean depth of flood tide decreases while those of ebb tide go opposite. Therefore, flow velocity of flood tide increases and that of ebb tide decreases, respectively. Sediment transport capacity of flood tide is enhanced relative to ebb tide due to the change of hydrodynamic characteristics, so that the sediment which is carried into the river by flood tide cannot be taken back to estuary by ebb tide, and then deposited in the lower reach downstream the floodgate.

\section{NUMERICAL MODEL AND THE CALCULATION SCHEMES}

To study the sediment characteristics in lower reach caused by floodgates in estuaries, a 2-D numerical model of the tidal currents and sediment transport is built based on the prototype of the floodgate in the estuary of the Sheyang River. Three different types of downstream floodgate are designed. The angle of direction of downstream floodgate and sea dynamic is $45^{\circ}, 90^{\circ}$ and $135^{\circ}$, 
respectively. The actual morphology of the estuary of the Sheyang River is simplified and applied as the open sea condition of the model. The open boundary of the model is about $12 \mathrm{~km}$ from the estuary, near the $-15 \mathrm{~m}$ contour. In addition, the area covered by the model between its South and North boundaries is about $21 \mathrm{~km}$ wide (Figure 1). In the model, the approach channel was simplified into a 10-kilometer-long and 660-meter-wide compound channel. The bottom of the inner passage of the approach channel is about 220 -meter-wide, with its elevation at $-9.0 \mathrm{~m}$. Side slope of the passage is $1: 8$, and the bottom elevation of the point bar is at about $0.0 \mathrm{~m}$.

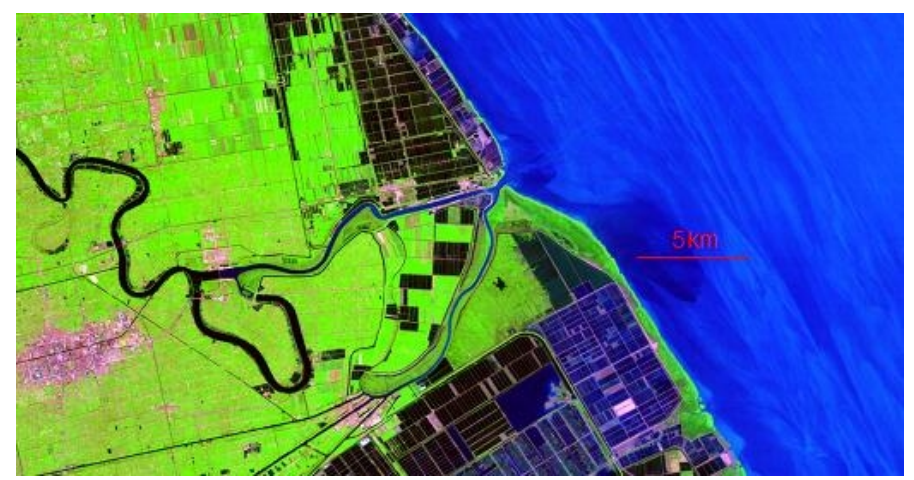

Figure 1. Domain of model boundary

The hydrodynamic boundary condition of the model is designed on the basis of the actual condition in the estuary of the Sheyang River. The high tide level is at $3.75 \mathrm{~m}$ and the low tide level is $0.75 \mathrm{~m}$ (Figure 2). The hydrodynamic condition of the open sea remains unchanged in the study. Figure 3 is the calculation topography and grid of the numerical model, the calculation grid number is $125 \times 101=12625$. The grids in lower reach of floodgates are refined, and the minimum feature size is $15 \mathrm{~m}$, the maximum feature size $400 \mathrm{~m}$ in coast outside.

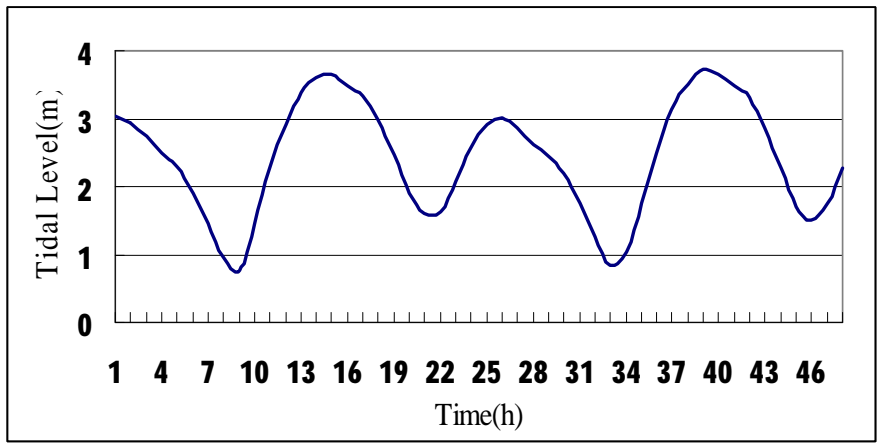

Figure 2. Design curve of tide on outer boundary

The continuity equation, momentum equation of tidal current and sediment transport equation in numerical model are discretized using FVM (Finite Volume Method):

$\frac{\partial \zeta}{\partial t}+\frac{\partial H u}{\partial x}+\frac{\partial H v}{\partial y}=0$

$\frac{\partial H u}{\partial t}+\frac{\partial H u u}{\partial x}+\frac{\partial H u v}{\partial y}=-g H \frac{\partial \varsigma}{\partial x}-\frac{\partial H \overline{u^{\prime} u^{\prime}}}{\partial x}-\frac{\partial H \overline{u^{\prime} v^{\prime}}}{\partial y}+\frac{\tau_{s x}-\tau_{b x}}{\rho}+f H v$

$\frac{\partial H v}{\partial t}+\frac{\partial H u v}{\partial x}+\frac{\partial H v v}{\partial y}=-g H \frac{\partial \varsigma}{\partial y}-\frac{\partial H \overline{v^{\prime}}}{\partial x}-\frac{\partial H \overline{v^{\prime}}}{\partial y}+\frac{\tau_{s y}-\tau_{b y}}{\rho}-f H u$

$\frac{\partial H S}{\partial t}+\frac{\partial H u S}{\partial x}+\frac{\partial H v S}{\partial y}=\frac{\partial}{\partial x}\left(H \varepsilon_{x} \frac{\partial S}{\partial x}\right)+\frac{\partial}{\partial y}\left(H \varepsilon_{y} \frac{\partial S}{\partial y}\right)+\alpha \omega\left(S_{*}-S\right)$

River bed evolution can be calculated by next formula: 
$\gamma_{0} \frac{\partial \eta_{s}}{\partial t}=\alpha \omega\left(S-S_{*}\right)$

Incipient motion estimate formula of cohesive sediment and sediment transport capacity of tidal currents and waves are key parameters in the numerical model of tide and sediment transport. Dou (1999) analyses the group characteristics and the movement characteristics of cohesive sediment, and deduced a calculation formula for starting velocity including bulk density effect, according to the effect of cohesive force between fine particles, water depth and bulk density etc.

$$
u_{c}=k^{\prime}\left(\ln 11 \frac{h}{\Delta}\left(\frac{\Delta}{\Delta_{*}}\right)^{1 / 6} \sqrt{3.6 \frac{\rho_{s}-\rho}{\rho} g d+\left(\frac{\gamma_{0}}{\gamma_{0}^{*}}\right)^{2.5} \frac{\varepsilon_{0}+g h \delta(\delta / d)^{1 / 2}}{d}}\right.
$$

Where $k^{\prime}$ is coefficient $\left(k^{\prime}=0.26\right.$ for impending motion, $k^{\prime}=0.32$ for little motion, $k^{\prime}=0.41$ for general motion), $\Delta$ is the roughness height of bed surface $(\Delta=1 \mathrm{~mm}$ for $\mathrm{d} \leq 0.5 \mathrm{~mm}, \Delta=2 \mathrm{~d}$ for $\mathrm{d}>0.5 \mathrm{~mm}), \Delta_{*}=20 \mathrm{~mm}, \rho_{S}$ and $\rho$ are the density of sediment particles and that of water respectively, $\gamma_{0}$ and $\gamma_{0}^{*}$ are the dry volume weight and the stable dry volume weight of the sediment on the bed surface. $\varepsilon_{0}$ is synthetic parameter of cohesive force whose value is related to the physical and chemical properties of the particle materials, and also to the organic content and siltation environment for cohesive soil, $\varepsilon_{0}=1.75 \mathrm{~cm}^{3} / \mathrm{s}^{2}$ for natural sediment. $\delta$ is the parameter of water film thickness, and $\delta=2.31 \times 10^{-5} \mathrm{~cm}$.

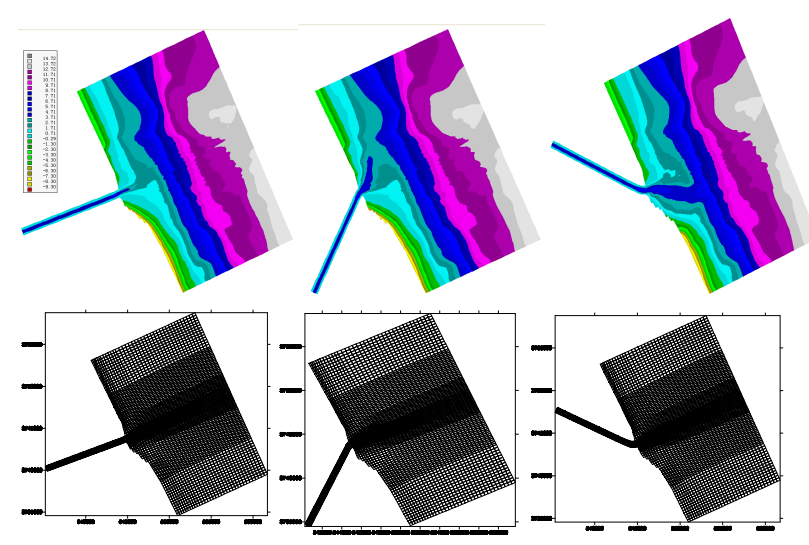

Figure 3. Calculation topography and grid of the numerical model

Luo (1997) deduced a calculation formula for sediment transport and carrying capacity for different wet densities of fluid mud, an overall verification of the calculation formula is made, and satisfactory results are obtained, showing that the calculation formula can be used as practical formula for the study of engineering-related sediment problems.

$S^{*}=0.296 \gamma_{s}\left(\frac{\gamma}{\gamma_{w}}\right)^{12.8} \frac{V^{2}}{g D}$

Where $\gamma_{w}$ is wet densities of fluid mud, $\gamma$ is densities of water, $\gamma_{s}$ is densities of sediment, $V$ is water velocity under the common action of tidal currents and waves, $D$ is water depth.

\section{THE SILTATION CHARACTERISTICS IN LOWER REACH CAUSED BY FLOODGATES}

The change curve of tidal level, current velocity and sediment concentration where is $4 \mathrm{~km}$ length downstream floodgates are shown in Figure 4. It shows that hydrodynamic characteristics changed after building floodgate include the duration and mean water depth of flood tide decreases, the averaged velocity of flood tide increases while those of ebb tide change in the opposite direction, and the high tide level is heightened while the low one goes lower. Flow velocity of flood tide increases 
and that of ebb tide decreases, respectively. Sediment concentration of flood tide is enhanced relative to ebb tide due to the change of hydrodynamic characteristics, so that the sediment which is carried into the river by flood tide cannot be taken back to estuary by ebb tide, and then deposited in the lower reach downstream the floodgate.

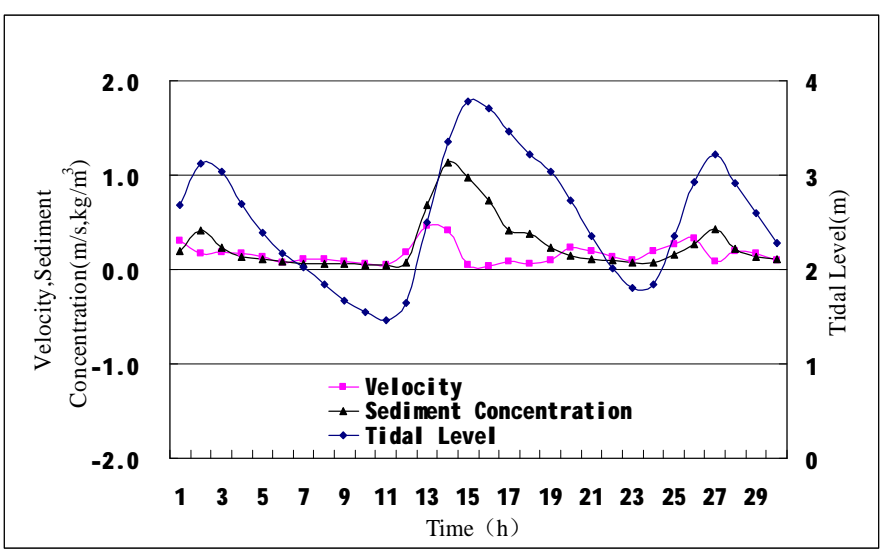

Figure 4. The change curves of tidal level, current velocity and sediment concentration

Figure 5 and Figure 6 show the distribution of sediment siltation downstream the floodgate which simulated by numerical model and the change of siltation volume, respectively. It can be found that deposition thickness near the floodgate is larger than siltation thickness in estuary, there is a slope from floodgate to estuary. The distribution characteristics of sediment siltation downstream the floodgate keeps consistent with the measured data.

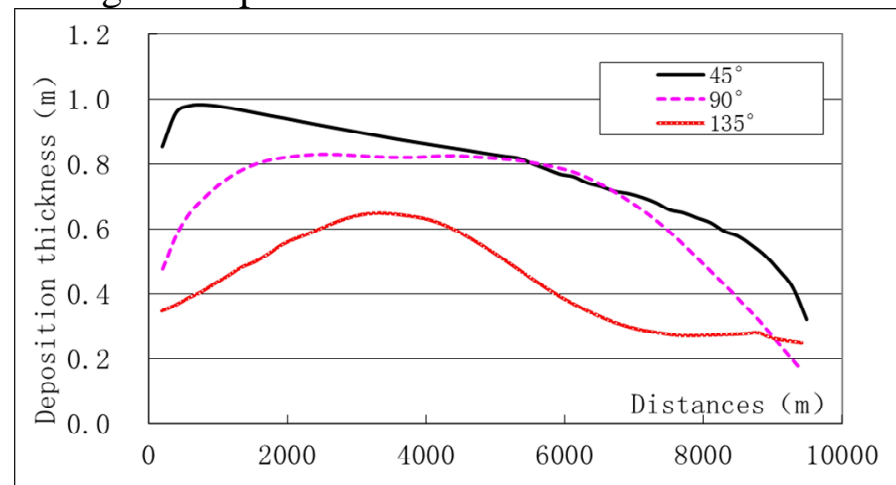

Figure 5. The distribution of sediment siltation downstream the floodgate (one year)

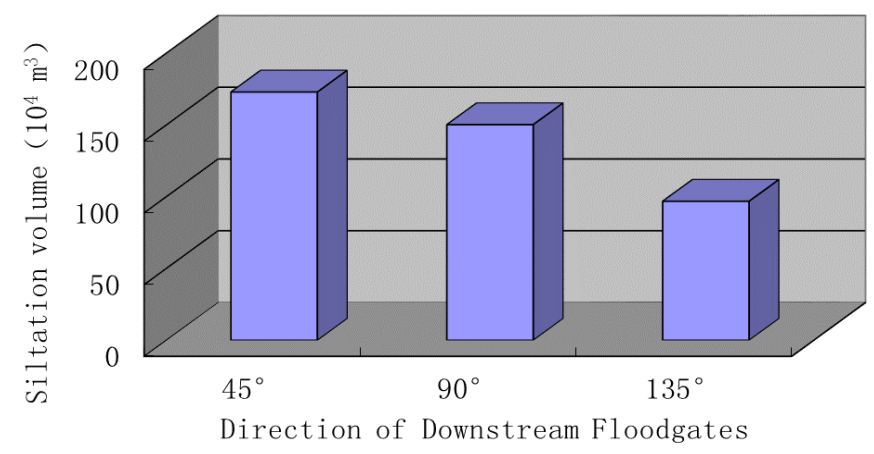

Figure 6 . The change of siltation volume 


\section{CONCLUSIONS}

(1) The simulation results of tide current and sediment transport numerical model show that tidal wave deformation after building floodgate makes the ratio increases of flood tide velocity and ebb tide velocity, and the sediment which is carried into the river by flood tide is larger than by ebb tide. Because coastal bottomland can provide enough sediment resource in the period of flood tide, while sediment deposited in lower reach of floodgates can't be eroded by ebb tide. The simulation results can reflect the processes of siltation downstream the floodgate in estuaries.

(2) According to the simulation results, the angle of direction of downstream floodgate and sea dynamic has great effect on the deposition downstream floodgates. When the angle is small, a large number of sediment are brought in by tide and the slope from floodgate to the estuary is large. The total siltation volume is large as well. As a consequence, the direction of downstream floodgates should be in correspondence with the ebb tide direction.

\section{ACKNOWLEDGEMENTS}

This work is supported by National Science Foundation (51409163) and National Sciencetechnology Support Plan Projects (2013BAB12B05-01)

\section{REFERENCE}

1)Dou Guoren, Analysis of Silting Problems under Sheyang River Floodgate. Collected Paper of Dou Guoren, Beijing: China Water Power Press (2003)

2) Xu Xuesong: Study on Siltation Characteristics in the Estuary with Sluices Constructed. Master Degree Dissertation, Hohai University (2012)

3) Luo Zhaosen: Study on Fluid Mud-Carrying Capacity, Sediment Transport and their Application, Journal of Sediment Research, Vol. 4, (1997), p.42-46

4) Dou Guoren: Incipient Motion of Sediment under Currents, China Ocean Engineering, Vol.14, (2000), p.391-416 


\title{
Study on influence of air decouple charge impact on blasting stress field in rock
}

\author{
Q Zong \& H B Wang \\ School of Civil Engineering and Architecture, Anhui University of Science and Technology, \\ Huainan, Anhui 232001
}

KEYWORD: Air; Decoupled charge; Blasting stress field; Experimental Investigation ABSTRACT: According to the theory of detonation and stress wave, initial shock pressure in wall of air-decoupled charge was deduced in theoretical and blasting stress field of air-decoupled charge in rock was established. Influence of decoupled coefficient to blasting stress field was analyses combined with test result, the result further proved decoupled charge can reduce compression stress obviously, the greater coefficient and stress reduce the more notable.

\section{INTRODUCTION}

At present, decoupled charge structure is one of widespread used charge structure; air and water are commonly used coupling medium, for the detonation wave and gas indirectly impact on blast-hole wall by through couplant, so compression force and blasting stress field in are different for difference between couplant. Both home and abroad also carried out many research in this area, mainly concentrated in blasting energy propagation of water and air decoupled, initial shock pressure, experimental investigation and numerical simulation of blasting mechanism ${ }^{[1 \sim 7]}$. However, theoretical study of air decoupled charge blasting stress field are seldom find, this article intended calculated blasting stress field of air-coupled charge in theoretical, and combined with the test results analysis influence of decoupled coefficient on blasting stress field.

\section{BLASTING STRESS FIELD CALCULATION OF AIR DECOUPLE CHARGE}

\section{CALCULATION OF HOLE WALL INITIAL SHOCK PRESSURE}

There are multiple calculation methods for air decoupled charge blasting hole wall the initial shock pressure calculation, basically have the following several:

1) Assumptions no air exist in the gap, explosion detonation products entropy expansion in gap with $k=3$, expansion initial pressure is average explosion detonation pressure, so evaluated hole wall initial impact pressure as follow ${ }^{[8]}$ :

$$
P_{b}=n P_{1}=\frac{n \rho_{e} D_{V}^{2}}{2(k+1)} \times K_{d}^{-2 k}
$$

Where: $P_{1}$ is explosion detonation products incident pressure before collision hole wall; $\rho_{e}$ is density of explosives; $D_{V}$ is detonation velocity of explosives; $K_{d}$ is decoupled coefficient of hole, $K_{d}=d_{b} / d_{c}, d_{b} 、 d_{c}$ is diameter of blasting hole diameter and charge respectively; $n$ is multiple of pressure increased when detonation gas impact hole wall, according to reference[8], $\mathrm{n}=8 \sim 11$.

2) Air exists between charge and hole wall, air shock wave generates in gap at first after explosive blast, then air shock wave impact on hole wall causes hole pressure, its value is:

$$
P_{b}=\frac{2 n}{\bar{k}+1} \times \rho_{a} D_{a}^{2}
$$

Where, $\rho_{a}$ is air density $\left(\rho_{a}=1.25 \times 10^{-3} \mathrm{~g} / \mathrm{cm}^{3}\right) ; D_{a}$ is air shock wave propagation velocity in hole wall, and related with charge decoupled coefficient and detonation products diffusion speed; $\bar{k}$ is average adiabatic index of air in gap, $\bar{k}=1.17 \sim 1.25 ; n$ still is multiple of pressure increased when detonation gas impact hole wall, but its value is different from preceding, $n=0 \sim 20$, exact value depends on incidence of air shock wave pressure. 
3) Above two methods are calculated by one-stage constant entropy expansion, which is different from the actual detonation gas state, especially at larger charge decoupled coefficient. So some studies suggest that, expansion process of detonation products should be considered as two-stage constant entropy expansion ${ }^{[9 \sim 11]}$, for average power industrial explosives, critical pressure of twostage demarcation point $P_{K}=200 \mathrm{MPa}$. When incidence of air shock wave pressure $P_{1} \geq P_{K}$, When incidence of air shock wave pressure $P_{1} \geq P_{K}$, calculated in accordance with k=3 constant entropy expansion, when $P_{1}<P_{K}$, calculated according to the $\gamma=1.3$ adiabatic expansion, so:

When, $\mathrm{P}_{1} \geq P_{K}$

$$
P_{b}=\frac{n \rho_{e} D_{V}^{2}}{2(k+1)} \times\left(\frac{d_{c}}{d_{b}+x}\right)^{2 k}
$$

When, $P_{1}<P_{K}$

$$
P_{b}=\left(\frac{P_{w}}{P_{K}}\right)^{\gamma / k} \times n P_{K} \times\left(\frac{d_{c}}{d_{b}+x}\right)^{2 \gamma}
$$

Where, $P_{1}$ is explosion detonation products incident pressure before collision hole wall; $P_{K}$ is critical pressure of two-stage demarcation point, its value is $200 \mathrm{MPa} ; n$ is multiple of pressure increased when detonation gas impact hole wall, general values is $8 ; \rho_{e}$ is density of explosive; $D_{V}$ is detonation velocity of explosive; $k$ is adiabatic index of detonation gas, $k=3 ; d_{b} 、 d_{c}$ is diameter of blasting hole diameter and charge respectively; $x$ is an instantaneous hole wall displacement; $\gamma$ is entropy of ideal gas, $\gamma=1.3 ; P_{w}$ is average detonation pressure, its value:

$$
P_{w}=\frac{\rho_{e} D_{V}^{2}}{2(k+1)}
$$

4) Because hole wall displacement is too small for negligible, in this condition, derived the following initial hole wall shock pressure formula of decoupled charge blasting ${ }^{[12]}$ :

$$
P_{r}=P_{d} \times K_{d}^{-2 k}=\frac{\rho_{e} D_{e}^{e}}{k+1} \times \frac{2 \rho_{m} C_{p}}{\rho_{m} C_{p}+\rho_{e} D_{e}} \times K_{d}^{-2 k}
$$

Physical meaning of symbol in the equation is the same as former. The formula adequately take influence of rock properties into consider, this is fundamental different from the above calculation method. In fact, extent of incident wave refraction and reflection at the hole wall is different for rock's impedance diversity.

\section{BLAST STRESS FIELD CALCULATION}

Put hole wall of the initial shock pressure of air-decouple charge into stress wave attenuation formula $^{[8]}$, access to blasting stress field of air-decoupled charge, that is,

$$
\sigma_{r}=\frac{P_{r}}{r}=\frac{p_{d} \times K_{d}^{-2 k}}{r}=\frac{\rho_{e} D_{e}^{e}}{k+1} \times \frac{2 \rho_{m} C_{p}}{\rho_{m} C_{p}+\rho_{e} D_{e}} \times K_{d}^{-2 k} \times\left(\frac{r}{r_{b}}\right)^{-\alpha}
$$

\section{BLASTING MODEL EXPERIMENT AND RESULTS ANALYSIS}

According to similarity theory and similarity criterion, small charge concrete model blasting experiment were did, tested the explosion stress in specimen, research the influence of decoupled coefficient to blasting stress field.

\section{MODEL PRODUCTION AND TESTING SYSTEM}

Model is poured by $425^{\#}$ Portland cement with sifted sand. To reduce the influence of boundary effect, we use rather larger sizes of model $350 \mathrm{~mm} \times 450 \mathrm{~mm} \times 700 \mathrm{~mm}$, maintenance more than 28 days. Strain brick use the same material and ratio as the test block, sizes is $30 \mathrm{~mm} \times 30 \mathrm{~mm} \times 300 \mathrm{~mm}$. Polish with sandpaper until it is smooth, then stick the strain gauge (BX120-5AA) with glue 502, its sensitivity coefficients is $2.08 \%$ and resistance $120 \Omega .8$ pieces for each strain brick, and layout a point every $60 \mathrm{~mm}, 4$ points totally. We welding on the single cable after the strain gages are sticked, insulating and waterproofing it with $\mathrm{AB}$ glue. 
Making use of the pre-receive strain brick as sensor to receive explosion signal, using super dynamic strain indicator and oscilloscope to collect and analyze the data. The super dynamic strain indicator are manufactured by Institute of Mechanics, Chinese Academy of Sciences, and the oscilloscope were manufactured by Agilent Technologies of United States, and the model number is 54815A, with bandwidth $500 \mathrm{MHz}, 4$ channels, sampling rate $1 \mathrm{GSa} / \mathrm{s}$.

Drilling fixed depth as $180 \mathrm{~mm}$ during the experiments, using special electric detonators (diameter of the detonator is $6 \mathrm{~mm}$, length of $40 \mathrm{~mm}$, installed $0.3 \mathrm{~g}$ dinitrodiazophenol, charge density of 1.0 $\mathrm{g} / \mathrm{cm}^{3}$, detonation velocity $4200 \mathrm{~m} / \mathrm{s}$, explosion heat $4000 \mathrm{~kJ} / \mathrm{kg}$ ). Small detonator installed to the end of hole, reserved air in the hole, and blocked off with fine sand and 502 glue, length of 40mm.

\section{Test results and analysis}

Test results shows in Table 1.

Table 1 Actual measurement results of radial stress

\begin{tabular}{cccccc}
\hline Hole diameter & Decoupled & \multicolumn{4}{c}{ Stress (MPa) } \\
\cline { 3 - 6 }$(\mathrm{mm})$ & coefficient & 1 & 2 & 3 & 4 \\
\hline 10 & 1.67 & 112.13 & 94.137 & 66.268 & 23.008 \\
12 & 2.00 & 99.089 & 75.716 & 41.618 & 16.918 \\
14 & 2.33 & 89.072 & 50.151 & 35.172 & 10.586 \\
16 & 2.67 & 61.424 & 47.506 & 13.429 & 7.105 \\
\hline
\end{tabular}

Curves of peak stress with proportional distance of different decoupled coefficient as Figure 1 and Figure2 shows; take testing data into formula (7), curves of calculated peak press with proportional distance as show in Figure3 and Figure4.

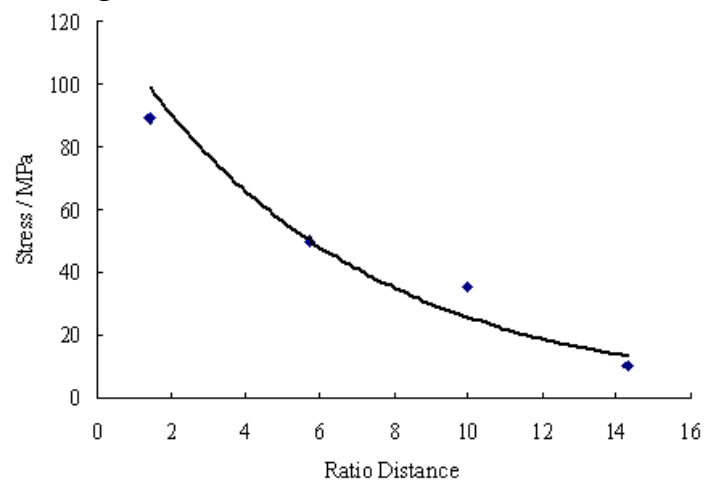

Figure1 The curve of peak pressure along with ratio distance $\left(K_{d}=2.33\right)$

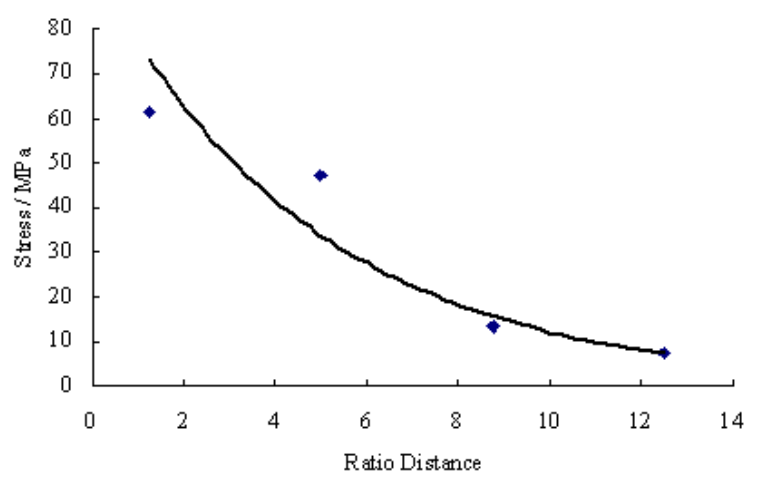

Figure2 The curve of peak pressure along with ratio distance $\left(K_{d}=2.67\right)$

According to testing and calculated results in Table 1, attenuation law of peak stress value with distance of air-decoupled charge blasting is: 


$$
\begin{aligned}
& K_{d}=1.67, \quad \sigma_{r}=295.83 \bar{R}^{-0.5524} \\
& K_{d}=2.00, \sigma_{r}=271.7 \bar{R}^{-0.664} \\
& K_{d}=2.33, \quad \sigma_{r}=243.15 \bar{R}^{-0.7779} \\
& K_{d}=2.67, \quad \sigma_{r}=182.52 \bar{R}^{-0.8915}
\end{aligned}
$$

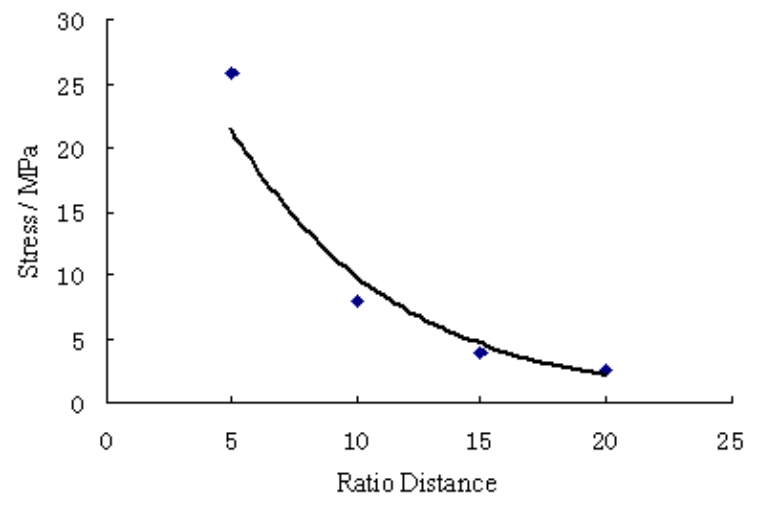

Figure3 The curve of stress along with ratio distance $\left(K_{d}=2.33\right)$

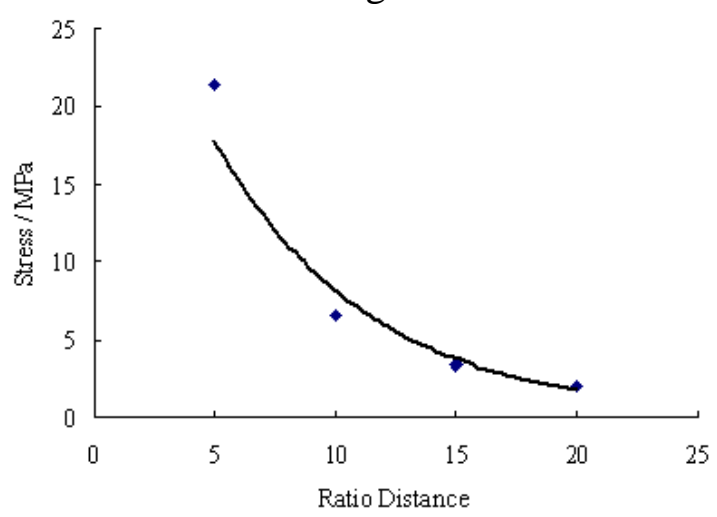

Figure4 The curve of stress along with ratio distance $\left(K_{d}=2.67\right)$

From blasting model test results and analysis conclusions can draw:

(1) The more near to the hole, the higher explosion stress, the peak stress decreased significantly as the distance increases, and decreasing rate of initial phase is larger, slow decline after the next stage, stress "platform phenomenon" appeared.

(2) With increment of decoupled coefficient $K_{d}$, the same measurement point's peak stress decreased, reaction time increased. With the increase of $K_{d}$ reflects explosives dynamic and static action relative change, its dynamic action weakening, static action strengthening. This is further proved the charge decouple coefficient does have significant influence on blasting pressure, air in the gap played a buffer role, The first explosive detonation wave compressed couple medium in the gap, forming a thin class of solid-phase material, a lot of elastic energy collected, so that part of the kinetic energy inverted, peak pressure caused by initial shock effect decreases and then, collected elastic energy released through the detonation gas, resulting "platform phenomenon", thus delaying the pressure attenuation, extended duration of action.

\section{CONCLUSIONS}

In this paper, blasting stress field calculation formula was deduced in theoretical, and influence of decoupled coefficient to blasting stress field was analyses combined with test result. The result proved that decoupled charge can reduce compression stress obviously, the greater coefficient and stress reduce the more notable. In actual blasting engineering, should according to engineering re- 
quirements, rock and construction conditions, choose rational charging structure, such as choose decouple charge structure with bigger decouple coefficient for presplit blasting, smooth, etc.

\section{ACKNOWLEDGEMENTS}

This research was financially supported by the National Natural Science Foundation (Grant No.51404010, 51374012, 51274009) and the Key Scientific and Technical Program of Anhui Province (No: 1501041123). The authors are grateful to the reviews for their valuable comments.

\section{REFERENCES}

[1] Zong Qi. Theoretical Analysis of Charge Structure's Influence on Pressure on Bore Wall [J]. Mining and Metallurgical Engineering, 2006, 26 (5): 9-13.

[2] Zong Qi, Luo Qiang. Experimental Study on Distribution Character of Blasting Stress When Boreholes with Water-Couple Charge [J]. Journal of Experimental Mechanics [J]. 2006, 21 (3): 393-397.

[3] Zong Qi, Meng Dejun. Influence of Different Kinds of Hole Charging Structure on Explosion Energy Transmission [J]. Chinese Journal of Rock Mechanics and Engineering, 2003, 22 (4): 641645.

[4] Du Junlin, Zhou Shengbing, Zong Qi. Theoretical analysis on pressure on bore wall of uncouple charge [J]. Journal of Xi ' an University of Science and Technology, 2007, 27 (3): 347-351.

[5] Wu Liang, Wei Min, Zhong Dongwang, et al.. Study on dynamic stress characteristics of airdecked charge explosion [J]. Blasting, 2009, 26 (4): 17-21.

[6] Wu Haijun, Yang Jun, HUANG Fenglei, et al.. Propagation behavior of stress wave of rock under the conditions of different coupling charges [J]. Mining Research and Development, 2002, 22 (1): 44-47.

[7] Jiang Pengfei, Tang Degao, Long Yuan. Numerical analysis of influence of uncoupled explosive-charge structure on stress field in hard rocks [J]. Rock and Soil Mechanics, 2009, 30 (1): 275279.

[8] Xu Ying, Zong Qi. Theory and Application of Underground Blasting [M]. Xuzhou: China Mining University Press, 2001.

[9] Gao Jinshi, Zhang Qi. Blasting Theory and Optimization[M]. Xi'an: Xi'an Map Press, 1993.

[10] Fei Honglu, Li Shouju, He Qingzhi. Determination of Decouple Coefficient and Analysis of Decouple Action in the Smooth Blasting. Explosion and Shock Waves, 1992, 12(3): 270-273.

[11] J.Henrych; Xiong Jianguo, et al.(Translation). The Dynamics of Explosion and Its Use [M]. Beijing: Science Press, 1987.

[12] Zhu Ruigeng, Wang Xuefeng, Decouple Charge Blasting Hole Wall Pressure Calculation [J]. Blasting, 1990, 7(3):1-3. 